\title{
Nonlinear Optics: The Enabling Technology for Quantum Information Science
}

\author{
Robert W. Boyd, ${ }^{1-3}$ Megan Agnew, ${ }^{1}$ Eliot Bolduc, ${ }^{1}$ Ebrahim Karimi, ${ }^{1}$ Jonathan Leach, ${ }^{1}$ \\ Omar S. Magana-Loaiza, ${ }^{2}$ Mehul Malik, ${ }^{2}$ Mohammad Mirhosseini, ${ }^{2}$ Malcolm N. \\ O'Sullivan, ${ }^{2}$ Jeff Z. Salvail ${ }^{1}$ and Zhimin Shi $^{2}$ \\ 1. Department of Physics, University of Ottawa, Ottawa, ON K1N 6 N5 Canada \\ 2. Institute of Optics, University of Rochester, Rochester, NY 14627 USA \\ 3.boydrw@mac.com
}

Nonlinear optical processes such as parametric down conversion and squeezed light generation are key elements of most quantum protocols, leading to crucial applications such as quantum imaging, sub-shot-noise metrology, and secure communication.

In this contribution, we argue that nonlinear optics is the key enabling technology for the blossoming field of quantum information science. The nonlinear optical processes of parametric down conversion and squeezed light generation are dominant processes in many quantum information protocols. From a different perspective, nonlinear optical effects such as self-action effects can modify the quantum statistics of light fields, leading either to advantageous or detrimental effects to various quantum protocols. Within this presentation, we illustrate these points through use of several examples.

One such example is afforded by the process of quantum imaging. Image formation making use of quantum states of light allows for dramatic new possibilities in the field of image science. We review some of the conceptual possibilities afforded by quantum imaging and describe some recent work that displays some of these features. We also address the complementary issue of how advanced imaging protocols can allow one to utilize and manipulate the enormous information content residing in the transvers structure of an optical field.

By its conventional definition, a photon is one unit of excitation of a mode of the electromagnetic field. The modes of the electromagnetic field constitute a countably infinite set of basis functions, and in this sense the amount of information that can be impressed onto an individual photon is unlimited. We describe how this large information content can be exploited for applications in quantum information science. As one example, we are currently developing a system to perform quantum key distribution at a high transmission rate by exploiting the transverse degree of freedom of the photon. Specifically, we aim to transmit more than one classical bit of information per photon by making use of this large information capacity.

More generally, we describe how image formation making use of quantum states of light allows dramatic new possibilities in the field of image science. One such example that we are studying is the possibility of performing imaging by impressing an entire image onto a single photon. We recently completed one study [1] that shows that by means of a holographic method we can discriminate between two objects even when they are illuminated by only a single photon. In a related study we have shown that we can discriminate among four objects using a single biphoton in a ghost-imaging configuration [2]. We have also studied $[3,4]$ the properties of light fields with transverse distributions that impart orbital angular momentum (OAM) onto the photon. These OAM states constitute a complete basis, and thus any quantum image can be described in terms of these states. Our work has quantified the thought that these states can be used as effective carriers of quantum information [5, 6].

We have also demonstrated that security protocols related to that of the BB84 protocol of QKD can be used to perform secure surveillance, that is, to ensure that image information in a scene of view has not been compromised [7]. Similar methods can be used for phase measurements in the field of quantum metrology. In one instance, we were able to establish a 16 -fold increase in phase sensitivity by combining quantum and classical protocols [8]. 
The final topic to be described is work aimed at the direct measurement of the quantum wavefunction. Historically, the wavefunction has often been considered to be primarily a conceptual entity that can be measured if at all using highly inefficient methods such as quantum tomography. However, Lundeen and his coworkers have recently shown [9] how by performing a "weak measurement" followed by a "strong measurement" it is possible to perform a measurement of the wavefunction in a direct and efficient manner. In recent work, my own group has demonstrated [10] that it similar methods can be used to measure directly the wavefunction of a qubit, which is the fundamental unit of information in quantum information science.

[1] C. J. Broadbent, P. Zerom, H. Shin, J. C. Howell, and R. W. Boyd Phys. Rev. A 79033802 (2009).

[2] M. Malik, H. Shin, M. O’Sullivan, P. Zerom and R. W. Boyd, Phys. Rev. Lett. 104, 163602 (2010).

[3] A. K. Jha, B. Jack, E. Yao, J. Leach, R.W. Boyd, G.S. Buller, S.M. Barnett, S. Franke-Arnold, and M.J. Padgett, Phys. Rev. A 78, 043810 (2008).

[4] J. Leach, B. Jack, J. Romero, M. Ritsch-Marte, R. W. Boyd, A. K. Jha, S. M. Barnett, S. Franke-Arnold, and M. J. Padgett, Opt. Express, 17, 8287 (2009).

[5] J. Leach, B. Jack, J. Romero, A. K. Jha, A. M. Yao, S. Franke-Arnold, D. G. Ireland, R. W. Boyd, S. M. Barnett, M. J. Padgett, Science 329, 662 (2010).

[6] M. Agnew, J. Leach, M. McLaren, F.S. Roux, R.W. Boyd, Phys. Rev. A 84062101 (2011).

[7] M. Malik, O.S. Magaña-Loaiza, and R.W. Boyd, Appl. Phys. Lett. 101, 241103 (2012).

[8] H. Shin, Omar S. Magaña-Loaiza, M. Malik, M.N. O’Sullivan, and R.W. Boyd, Optics Letters 21, 2816 (2013).

[9] J.S. Lundeen, B. Sutherland, A. Patel, C. Stewart, C. \& C. Bamber, Nature 474, 188-191 (2011).

[10] J.Z. Salvail, M. Agnew, A.S. Johnson, E. Bolduc, J. Leach and R.W. Boyd, Nature Photonics, DOI: 10.1038/NPHOTON.2013.24 (2013). 